\title{
Gas Turbine Condition Monitoring and Prognosis: A Review
}

\section{Shasha Wang ${ }^{1, a}$, Weimin Wang ${ }^{1, b}$, Yongqiang Shi ${ }^{1, c}$, Ya Zhang ${ }^{1, d}$}

\author{
${ }^{1}$ Diagnosis and Self-recovery Engineering Research Center, Beijing University of Chemical \\ Technology, Beijing 100029, China \\ asusan202020@163.com, bwwmbuct@163.com, 'shiyq1987@gmail.com \\ dzhangya@mail.buct.edu.cn
}

Keywords: gas-turbine, condition monitoring, fault diagnosis, prognosis

\begin{abstract}
Gas-turbine engines are critical to the operation of most industrial plants, aircraft and heavy vehicles. Condition monitoring is essential to mastering mechanical system running status, improving operation reliability, and reducing maintenance cost. This paper reviews state-of-the-art gas turbine condition monitoring, puts forward the pending problems and predicts future development in the field. Three main advanced methods are introduced briefly in the end.
\end{abstract}

\section{Introduction}

Gas-turbine engines are critical to the operation of most industrial plants, aircraft, and heavy vehicles such as military armour and transport ships, and their associated maintenance costs can be high. Because of complex structure and poor working conditions, it becomes the most sensitive part in whole system. Condition monitoring is essential to mastering mechanical system running status and the function characteristic and it also play an important role in avoiding breakdown and hazardous accidents, improving operation reliability, and reducing maintenance cost. By adopting a condition monitoring approach, the engine's maintenance needs are determined according to its operating condition, rather than being performed at fixed periods of time ${ }^{[1]}$.

\section{Current Research at Home and Abroad}

Condition monitoring and prognosis for gas-turbine is to detect fault (failure) or anomaly conditions, pinpoint or isolate which component/object in a system/ process is faulty, and decide on the potential impact of a failing or failed component on the health of the system. Researchers in such diverse disciplines as medicine, engineering, the sciences, business, and finance have been developing numerous methods over the past decades. NASA represents the highest level of turbine machinery. Refs. [2] researches in health monitoring of turbine components based on original vibration measurement and do some experimental research. Refs. [3] Presents a method of health monitoring for gas turbine combined theory with experiment Refs.[4] measures clearance between the top of turbine blades and shell using capacitive displacement sensor to detect cracks appeared on the turbine disc, and experiment was investigated in a high-speed rotor test-bed. Refs. [5] puts forward an active clearance control system, and points out that the measurement of clearance in blade tip is the key problem of this technique. Refs. [6] proposes a measurement of clearance in blade tip based on microwave and does some experimental research. It is proved have good prospects. 
In general, there are four existing techniques for condition monitoring and prognosis of gas-turbine engines ${ }^{[1]}$ :

\section{1) Standard Methods ${ }^{[7,8]}$}

Guidelines for the production of condition monitoring systems applied to aircraft gas-turbine engines have been available for over twenty years. Engine monitoring is performed using either "on-line" system, mounted within the aircraft, that perform analysis of engine data during flight, or "off-line" ground-based systems, to which engine data is downloaded from the aircraft at the end of a flight. Current research on the condition monitoring of aircraft engines is focused on providing more sophisticated methods of reliably determining engine condition and resulting maintenance recommendations, within the constraints of on-line and off-line monitoring systems.

2) Fault Diagnosis using Expert Systems ${ }^{[9,10]}$

Expert systems aim to capture the knowledge of domain experts, typically from engine manufacturers, in order to diagnose faults from engine data and recommend appropriate maintenance actions. The accuracy of diagnosis may be improved through the construction of ever-larger databases of fault information for gas-turbine engines. CASE-based Reasoning (CBR) uses the intuition that solutions to new problems are likely to be similar to previously encountered problems of a similar nature. However, the CBR approach is limited by the variation in operational behavior between classes of aircraft engine, and even variation between engines of the same class, which can render the use of general fault databases ineffective when diagnosing engine-specific faults. The combination of expert system databases from multiple engines of the same class has been attempted, aiming to generate systems that might generalize to other engines of that class.

\section{3) Thermo dynamical and Frequency-based Modeling ${ }^{[11-13]}$}

Thermo dynamical models of gas-turbine engines have been used for condition monitoring by comparison of a model derived from actual engine data (usually temperatures and pressures) with a model constructed from the theoretical relationship between those engine data. The residual error between the two models may be used to identify possible engine failures. Frequency-domain approaches to modeling engine parameters have been used in fuel-flow monitoring, exhaust gas monitoring, and more general diagnosis of engine faults. These models have been combined with auto-regressive, moving-average (ARMA) models for fuel-flow monitoring, and for analysis of engines used in industrial plants.

\section{4) Statistical Pattern Recognition and "Soft Computing" Approaches ${ }^{[14,15]}$}

Determining engine condition by comparison of engine data with a model of normal behavior may also be achieved using "soft computing" methods of model creation, such as neural networks, or using statistical pattern recognition techniques. These methods offer the advantage that engine condition may be assessed without detailed theoretical system models. These data-driven approaches can be used to construct models of engine behavior directly from engine data, in contrast to rule-based approaches that encode expert knowledge, and may be adapted for use with individual engine units, providing engine-specific condition monitoring. However, these methods can be sensitive to the quality and quantity of example data from which models of engine operation are constructed. Neural networks have been used to identify abnormalities in the operation of gas-turbine engines within industrial plants, in space shuttle engines, and in engines used by heavy ships. The support vector machine (SVM) method, a statistical pattern recognition approach, has been used to identify examples of abnormal engine vibration behavior from abnormal training set in aircraft engine production tests, and in water-pump gas-turbine engines. These one-class classifiers learn a model of normality from a training set of normal examples, and identify patterns that fall outside that class as abnormal, in comparison with multi-class classifiers, which require 
approximately equal numbers of examples for each class. Other methods of constructing models of normal engine behavior within engine production tests using data-driven techniques have been successfully demonstrated using data from military aircraft engines. Automated extraction of patterns describing normal and abnormal gas turbine engine operating conditions with engine vibration data has been investigated set within a general fuzzy-neural analysis framework.

\section{Development}

At present, the most famous monitoring systems for gas turbine over the world are: TEAM III from PW in United States, GEM from GE, COMPASS from Rolls-Royce(RR) in Britain, and the function of them are usually: 1) Performance monitoring and fault diagnosis. Analyze performance level and performance recession degree of gas turbine parts mainly through monitoring total temperature, total pressure, static pressure, rotor speed and fuel flow of the main section; 2) Mechanical condition monitoring and fault diagnosis. Monitor the healthy state of gas turbine system mainly through measurement of vibration data and oil parameters, testing of chips contained in lube oil and analysis of the results;4) Non-destructive testing and experiment. Detect mechanical damage by using special equipment. In general, four general subject which should be concentrated on during detecting: 1) the thermodynamic gas path, 2) vibration of bearings, rotors, etc., 3) lubrication, and 4) controls, summarizes the characteristics of various monitoring methods and their applications.

Prognosis is the ability to predict accurately and precisely the remaining useful life of a failing component or subsystem. In the industrial and manufacturing arenas, prognosis is interpreted to answer the question, "What is the remaining useful lifetime of a machine or a component once an impending failure condition is detected, isolated, and identified?'It is the crucial problem of the condition-based maintenance/prognostic health management system and presents major challenges to system designer primarily because it entails large-grain uncertainty. Accurate and precise prognosis demands good probabilistic models of the fault growth. Physics-based models have been employed extensively to represent the initiation and propagation of structural anomalies. Model-based prognostic schemes employ a dynamic model of the process being predicted, and provide a technically comprehensive approach that has been used traditionally to understand component failure mode progression. Implementing physics-based models can provide a means to calculate the damage to critical components as a function of operating conditions and assess the cumulative effects in terms of component life usage.

Intelligent Control and Health Monitoring with advanced model-based control architecture overcomes the limitations of state-of-the-art engine control and provides the potential of virtual sensors. Currently, health monitoring units are stand-alone monitoring units. Adaptive models open up the possibility of adapting the control logic to maintain desired performance in the presence of engine degradation. The key enabling technologies for an intelligent condition monitoring system are the increased efficiencies of components through active control, advanced diagnostics and prognostics integrated with intelligent engine control to enhance operational reliability and component life, and distributed control with smart sensors and actuators in an adaptive fault tolerant architecture. Development of appropriate sensors and actuation systems is critical to affordable and reliable implementation of the technologies for a more intelligent gas turbine engine. Improved and new sensors are required to allow:1) Sensing at stations with high operating temperatures; and2) Additional monitoring of vibration, mass flows, fuel properties, exhaust gas composition, and gas path debris ${ }^{[16]}$. 


\section{Three New Methods in Condition Monitoring and Prognosis}

\section{1) Measurement of Blade Tip Clearance}

The active control and minimization of the gap between the rotating turbine blades and the stationary case of gas turbine engines is being investigated as a means of increasing engine efficiency, reducing fuel consumption, reducing emissions, and increasing engine service life ${ }^{[17]}$. Research in modern gas turbine of Rolls-Royce (RR) company shows that engine efficiency decrease $1.5 \%$ as tip clearance increase $1 \%$ of blade length; and fuel consumption increase $2 \%$ as engine efficiency decrease 1\%. According to analysis of CF5-50 engine from GE, tip clearance accounts for $67 \%$ of total loss in fuel consumption. So, tip clearance should be tried to minimize in design, but too small gap may influence the engine's safety ${ }^{[18,19]}$. NASA Glenn research center's rotordynamics laboratory monitor the crack on turbine disk by using tip clearance data, and have made great progress ${ }^{[20]}$. The theoretical basis is that if there was a crack on the turbine disk, the deformation will be caused by this flaw under working condition, and the centroid of disk will change slightly with rotor speed. we can use measurement of tip clearance and shaft vibration to monitor centroid changes and blade deformation of rotating system. Foreign aviation research institutes have developed many methods of clearance measurement, some of the most commonly used are: discharge probe (spark discharge), the eddy current, capacitive probe ${ }^{[21.22]}$, fiber, et.

\section{2) Analysis of oil sample}

Engine condition monitoring system is mainly used to prevent early fault of component such as engine bearings, gears, rotating seal, and to monitor physical quality of lubricant. Compared with other kinds of analysis technology, oil analysis technology is reliable, less environmental disturbance, and neither need neither disassembling nor limited by onboard monitoring parameters. This new method is attracting great interests from researchers all over the world and developing rapidly. At present, oil analysis technology mainly have three methods: 1) Performance analysis of lubricant. The current widespread method of mechanical equipment lubrication monitoring is to judge mechanical failure through analyzing the change of lubricant's physical-chemical properties and degradation. 2) Pollution analysis. Analyze the level of environmental pollution. 3) Analysis wearing particle. Determine the wear state, degree, and type through wear monitoring based on the analysis of lubricant contamination ${ }^{[23,24]}$. Commonly used oil analysis technology is: dielectric constant method ${ }^{[25]}$, Fourier transform infrared spectroscopy ${ }^{[26]}$, lubricant monitoring technology based on image recognition ${ }^{[27]}$, ferrous spectrum technology.

\section{3) Stress wave technology}

Early in the 1960s, the stress wave technology were used in some structure detection. The principle is to use the transmission time, speed and waveform conversion of stress wave in the component to predict the mechanical properties and internal structure of tested components, then judge its use status. Refs.[28] proposed that the stress wave and wavelet monitoring are the most effective methods for bearing monitoring of large turbine in The Eight International Conference on Electrical Machines and Systems(ICEMS 2005). At present, some mature methods of fluctuation numerical solution in application are: wave function expansion method, the characteristic line method, finite element method and the wavelet transform method, etc., which were used in different specific situation. Further research in constitutive relationship between stress wave and material and building the function relation between internal fault and external measurement are important research direction in condition monitoring and prognosis. 


\section{Conclusion}

Gas-turbine engines are critical to the operation of most industrial plants, aircraft and heavy vehicles, and their associated maintenance costs can be high. Researchers in such diverse disciplines as medicine, engineering, the sciences, business, and finance have been developing numerous methods over the past decades. Existing techniques for condition monitoring of gas-turbine engines include traditional methods, fault diagnosis using expert systems, thermo dynamical and frequency-based modeling, statistical pattern recognition and "soft computing" approaches. On the basis of research on the current different condition monitoring system equipped in the current turbine machinery, presents that research on fault mechanism and sensor technology can enhance the intelligent system do more of the analysis and decision making, while continuing to support the depth of analysis currently available at experienced operations. Improved and new sensors are required to allow: 1) sensing at stations with high operating temperatures; and 2) additional monitoring of vibration, mass flows, fuel properties, exhaust gas composition, and gas-path debris. Finally, three main advanced methods are introduced briefly in the end, which are condition monitoring based on measuring Blade tip clearance, analysis of lubricant contamination and stress wave analysis.

\section{Acknowledgments}

This work was supported by the general Program of Natural Science Foundation of China (50975018) and the Fundamental Research Funds for the Central University(ZD0903).

\section{Reference}

[1] David Clifton. Condition Monitoring of Gas-Turbine Engines, D. Department of Engineering Science, University of Oxford, 2006.

[2] Andrew L. Gyekenyes, Jerzy T. Sawicki, Richard E. Martin. Vibration Based Crack Detection in a Rotating Disk, NASA/TM-2005-212624.

[3] Ali Abdul-Aziz, Mark R. Woike, John D. Lekki, George Y. B aaklini. Health Monitoring of a Rotating Disk Using a Combined Analytical-Experimental Approach. NASA/ TM-2009-21675.

[4] Mark R. Woike, Ali Abdul-Aziz. Crack-detection Experiments on Simulated Turbine Engine Disks in NASA Glenn Research Center's Rotordynamic Laboratory. NASA/ TM-2010-216239.

[5] Mark R. Woike, Ali Abdul-Aziz. Crack-detection Experiments on Simulated Turbine Engine Disks in NASA Glenn Research Center's Rotordynamic Laboratory. NASA/ TM-2010-216239.

[6] Mark R. Woike, James W. Roeder, Christopher E. Hughes, et al. Testing of a Microwave Blade Tip Clearance Sensor at the NASA Glenn Research Center. NASA/TM-2009-215589.

[7] SAE. Aircraft Gas Turbine Engine Monitoring System Guide. Technical report, Aerospace Recommended Practice, 1981.

[8] SAE. A Guide to Aircraft Turbine Engine Vibration Monitoring Systems. Technical report, Aerospace Information Report, 1986.

[9] Pena JM, Famili F, Letourneau S. Data Mining to Detect Abnormal Behavior in Aerospace Data. In KDD, 2000. 
[10] Trammel C, Vossler G, Feldmann M. UK Ministry of Defence Generic Health and Usage Monitoring System. American Helicopter Society Annual Forum, 3, 1997.

[11] Booth C, McDonald JR, Donald P, Lines N, Cooke N, Smith C. Model-based Condition Monitoring of Gas Turbine Engines for Power Generation Duty. IEEE Power Engineering Review, 2001.

[12] Evans C, Rees D, Hill D. Frequency Domain Identification of Gas Turbine Dynamics. IEEE Transactions on Control Systems Technology, 1998.

[13] Simani S. Identification and Fault Diagnosis of a Simulated Model of an Industrial Gas Turbine. IEEE Transactions on Industrial Informatics, 2005.

[14] Meyer CM, Maul WA, Dhawan AP. SSME Parameter Estimation and Model Validity Using Radial Basis Function Neural Networks. In Proc. of the 1994 Conf. on Advanced Earth to Orbit Propulsion Technology, 1994.

[15] Duda RO, Hart PE, Stork DG. Pattern Classification. Wiley, 2 edition, 2001.

[16] Dennis Gulley, Sanjay Garg, S.J. Hiller, Wolf Gang Hom, et al. More Intelligent Gas Turbine Engines. RTO-TR-AVT-128, April 2009.

[17] Mark R. Woike, James W. Roeder, Christopher E. Hughes, and Timothy J. Bencic. Testing of a Microwave Blade Tip Clearance Sensor at the NASA Glenn Research Center, NASA/TM-2009-215589.

[18] Na Zhang, Chunfeng Huang. Measuring Technology of Blade Tip Clearance of Aeroengine, J. Aeronautical Manufacturing Technology. 13(2010) 41-45.

[19] J.W.H.Chivers, A Technique for the Measurement of Blade Tip Clearance in a Gas Turbine, AIAA, 1989, 89, 2916 2921.

[20] Mark R. Woike, Ali Abdul-Aziz. Crack-Detection Experiments on Simulated Turbine Engine Disks in NASA Glenn Research Center's Rotordynamics Laboratory, NASA/TM-2010-216239.

[21] Alexander Steiner. Techniques for blade tip clearance measurements with capacitive probes, J. Measurement Science and Technology. 11 (2000) 865-869.

[22] D. Muller. Capacitive of Compressor and Turbine Tip to Cunning Clearance. ASME 96-GT-349, 1996.

[23] Jinglin Wang, Yanjun Li, Yibin Lu, et al. Recent Advancement in Aero-Engine Health Monitoring, J. Aircraft Design. 3 (2009) 56-60.

[24] Xinfa Shi, Dongfeng Liu. Development of Decay Monitoring Technique for Lubrication Oils, J. China Plant Engineering. 6 (2007) 47-48.

[25] Kai Liu. Oil Monitoring Method Based on the Dielectric Constant, J. Lubrication Engineering. 1 (2009) 94-96.

[26] Huangqing Hu. Techniques for blade tip clearance measurements with capacitive probes, J. Wood Processing Machinery.15(2004)44-49.

[27] Yong Zhang, Ruixiang Zhu, Shuichang Liu, et al. Monitoring for Lubricant Debris Using Image Manipulation Technique, J. Tractor \& Farm Transporter. 4 (2008) 91-93.

[28] Changzheng Chen, Changcheng, Yu Sun Zhang, et al. Fault Diagnosis for Large-scale Wind Turbine Rolling Bearing Using Stress Wave and Wavelet Analysis, R. Shenyang, 2005. 